\title{
Effect of molecular bending on the photodissociation of OCS
}

\section{$\operatorname{AUTHOR}(\mathrm{S}):$}

Sugita, A; Mashino, M; Kawasaki, M; Matsumi, Y; Bersohn, R; Trott-Kriegeskorte, G; Gericke, KH

\section{CITATION:}

Sugita, A ... [et al]. Effect of molecular bending on the photodissociation of OCS. JOURNAL OF CHEMICAL PHYSICS 2000, 112(16): 7095-7101

\section{ISSUE DATE:}

2000-04-22

URL:

http://hdl.handle.net/2433/39724

\section{RIGHT:}

Copyright 2000 American Institute of Physics. This article may be downloaded for personal use only. Any other use requires prior permission of the author and the American Institute of Physics. 


\title{
Effect of molecular bending on the photodissociation of OCS
}

\author{
Akihiro Sugita, Michio Mashino, and Masahiro Kawasaki \\ Department of Molecular Engineering, Kyoto University, Kyoto 606-8501, Japan \\ Yutaka Matsumi \\ Solar-Terrestrial Environment Laboratory, Nagoya University, Toyokawa 442-8507, Japan \\ Richard Bersohn \\ Department of Chemistry, Columbia University, New York, New York 10027 \\ Gundula Trott-Kriegeskorte and Karl-Heinz Gericke \\ Institut fur Physikalische und Theoretische Chemie, Technische Universitat Braunschweig, \\ Hans-Sommer-Strasse 10, D-38106, Braunschweig, Germany
}

(Received 4 January 2000; accepted 31 January 2000)

\begin{abstract}
At $230 \mathrm{~nm}$, the photodissociation of OCS via a hot band and a triplet state was investigated by selective probing of high rotational levels of product $\mathrm{CO}(J=45-67)$ with photofragment imaging spectroscopy: $\operatorname{OCS}(v=0)+h \nu(\mathrm{UV}) \rightarrow \mathrm{CO}\left(X^{1} \Sigma^{+}, J \geqslant 65\right)+\mathrm{S}\left({ }^{3} P\right), \quad \mathrm{OCS}(v=1)+h \nu(\mathrm{UV})$ $\rightarrow \mathrm{CO}\left(X^{1} \Sigma^{+}, J \sim 65\right)+\mathrm{S}\left({ }^{1} D\right)$. Additional two-photon IR excitation of the UV photoprepared OCS with intense $1.06 \mu \mathrm{m}$ laser pulses bleaches the UV processes listed above and induces a new excitation channel of OCS: $\mathrm{OCS}(v=0)+h \nu(\mathrm{UV}) \rightarrow \mathrm{OCS}^{*}, \quad \mathrm{OCS}^{*}+2 h \nu(\mathrm{IR}) \rightarrow \mathrm{CO}\left(X^{1} \Sigma^{+}, J\right.$ $\sim 74)+\mathrm{S}\left({ }^{1} S\right)$. The bending mode of OCS in the excited states plays a central role in the excitation and dissociation dynamics. Additionally, the alignment effect of OCS by nonresonant infrared laser pulse, which appears on the angular distribution of the photofragment, is discussed. (C) 2000 American Institute of Physics. [S0021-9606(00)00316-0]
\end{abstract}

\section{INTRODUCTION}

OCS, like all triatomic molecules with 16 valence electrons, is linear in its ground state and bent in its first excited state. It serves as a model for the effect of bending on photodissociation dynamics. When OCS is excited in its first UV band, singlet channel (1) is the main dissociation pathway that produces rotationally hot but vibrationally cold $\mathrm{CO}$ molecules along with $\mathrm{S}\left({ }^{1} D\right)$ atoms: ${ }^{1-4}$

$$
\begin{aligned}
\operatorname{OCS}(v=0)+h \nu(\mathrm{UV}) & \rightarrow \mathrm{OCS}\left(1^{1} \Delta\left(2^{1} A^{\prime}\right)\right) \\
& \rightarrow \mathrm{CO}\left(X^{1} \Sigma^{+}, J\right)+\mathrm{S}\left({ }^{1} D\right) .
\end{aligned}
$$

The doubly degenerate $\operatorname{OCS}\left(1^{1} \Delta\right)$ splits into two nondegenerate states upon bending, $\operatorname{OCS}\left(2{ }^{1} A^{\prime \prime}\right)$ and $\operatorname{OCS}\left(2{ }^{1} A^{\prime}\right)$. The $2{ }^{1} A^{\prime}$ state has a deep minimum in the bending mode potential and an equilibrium bond angle of $\sim 130^{\circ}$. This state carries most of the transition intensity of the first UV transition. ${ }^{3}$ A small portion of the UV absorption intensity is due to the $1^{1} A^{\prime \prime}\left(1^{1} \Sigma^{-}\right)$state that has a minimum energy structure similar to that of the $2{ }^{1} A^{\prime}\left(1^{1} \Delta\right)$ state. These potential curves are shown schematically in Fig. 1. Because the two bent states are involved in the UV dissociation, most of the available energy is allocated to the rotational motion of CO. Its rotational distribution is bimodal mainly due to two different processes on the $2{ }^{1} A^{\prime}\left(1^{1} \Delta\right)$ surface, direct dissociation and surface crossing to the ground state. ${ }^{3}$

In spite of the large spin-orbit interaction terms in $\mathrm{S}$ and $\mathrm{O}$ atoms, triplet channel (2) was reported to be $5 \%$ relative to singlet channel (1) at $222 \mathrm{~nm} .{ }^{5}$ The energy levels of the lowest triplet states $\operatorname{OCS}\left({ }^{3} A^{\prime}\right.$ and $\left.{ }^{3} A^{\prime \prime}\right)$ were calculated to be $\sim 3.5 \mathrm{eV}$ at an equilibrium bond angle $=123^{\circ}$ and the vertical energy was measured to be $4.94 \mathrm{eV}$, respectively. ${ }^{6,7}$ These potential surfaces may intersect with the photoprepared singlet states in the bend coordinate to open a triplet dissociation channel. In this work we have investigated a triplet dissociation channel by probing highly rotationally excited $\mathrm{CO}$ :

$$
\begin{aligned}
\mathrm{OCS}+h \nu(\mathrm{UV}) & \rightarrow \mathrm{OCS} *(\text { singlet }) \rightarrow \mathrm{OCS}(\text { triplet }) \\
& \rightarrow \mathrm{CO}\left(X^{1} \Sigma^{+}, J\right)+\mathrm{S}\left({ }^{3} P\right)
\end{aligned}
$$

In a cold molecular beam, no hot band dissociation has been reported for OCS. However, for example, the hot band dissociation channel of $\mathrm{O}_{3}\left(\nu_{\text {bent }}=1\right)$ to $\mathrm{O}\left({ }^{1} D\right)+\mathrm{O}_{2}\left({ }^{1} \Delta\right)$ in the Huggins band has been found even in cold molecular beam, which is an additional source of $\mathrm{OH}$ radicals in troposphere chemistry. ${ }^{8}$ The UV photoabsorption of $\mathrm{N}_{2} \mathrm{O}$ is greatly increased by excitation of the bending mode. ${ }^{9}$ Thus, any hot band channel at the longer UV region should be open for bent OCS due to energy lowering of the electronic transitions and enhanced Franck-Condon factors upon bending:

$$
\mathrm{OCS}\left(v_{2}=1\right)+h \nu(\mathrm{UV}) \rightarrow \mathrm{CO}\left(X^{1} \Sigma^{+}, J\right)+\mathrm{S}\left({ }^{1} D\right) .
$$

In the vacuum UV absorption band, $\mathrm{S}\left({ }^{1} D\right)$ and $\mathrm{CO}$ are produced from the photodissociation via direct VUV excitation of the $\operatorname{OCS}\left({ }^{1} \Sigma^{+} \leftarrow X^{1} \Sigma^{+}\right)$transition. ${ }^{10}$ At $154-157 \mathrm{~nm}$ the partition of internal and translational energy was investigated for this repulsive state. ${ }^{11,12}$ Due to the linear structure of OCS and stiffness of the $\mathrm{C}-\mathrm{O}$ bond, (a) most of the available energy is allocated to relative translational motion, and (b) the angular anisotropy is as high as $\beta=1.8 \pm 0.2$. 
If a stepwise transition through the UV prepared (OCS $\left(1{ }^{1} \Delta\left(2{ }^{1} A^{\prime}\right)\right)$ occurs by multiphoton absorption to the VUV energy region, the dissociation pathway may be controlled by adjusting the pumping rate to the upper dissociative state. In this work, we have prepared the upper repulsive state by $1.06 \mu \mathrm{m}$ excitation through the UV photoprepared repulsive state $\operatorname{OCS}\left(1^{1} \Delta\left(2{ }^{1} A^{\prime}\right)\right)$. Complete switching of dissociation pathway (1) to pathway (4) is achieved by controlling the intensity of the IR laser:

$$
\begin{aligned}
\operatorname{OCS}(v=0) & +h \nu(\mathrm{UV})+2 h \nu(\mathrm{IR}) \\
& \rightarrow \mathrm{CO}\left(X^{1} \Sigma^{+}, J\right)+\mathrm{S}\left({ }^{1} S\right) .
\end{aligned}
$$

\section{EXPERIMENT}

A pulsed YAG pumped dye laser (5 ns, $0.1-0.2 \mathrm{~mJ} /$ pulse at UV, $10 \mathrm{~Hz}$ ) frequency doubled to $229.83-230.00$ $\mathrm{nm}$ was used to dissociate OCS in a pulsed molecular beam. Photofragment $\mathrm{CO}(J=45-67)$ or $\mathrm{S}\left({ }^{1} D\right)$ were ionized by the same UV laser light via $(2+1)$ resonance-enhanced multiphoton ionization (REMPI). The following processes occur:

Dissociation

$$
\mathrm{OCS}+h \nu(\mathrm{UV}) \rightarrow \mathrm{CO}\left(X^{1} \Sigma^{+}, J\right)+\mathrm{S}\left({ }^{1} D\right) .
$$

\section{REMPI of CO}

$$
\begin{aligned}
& \mathrm{CO}\left(X^{1} \Sigma^{+}, J\right)+2 h \nu(229.84-229.98 \mathrm{~nm}) \\
& \rightarrow \mathrm{CO}\left(B^{1} \Sigma^{+}, Q \text {-branches }\right) \\
& \mathrm{CO}\left(C^{1} \Sigma^{+}\right)+h \nu(\mathrm{UV}) \rightarrow \mathrm{CO}^{+} .
\end{aligned}
$$

REMPI of $\mathrm{S}\left({ }^{1} D\right)$

$$
\begin{aligned}
& \mathrm{S}\left(3 p^{41} D\right)+2 h \nu(230.00 \mathrm{~nm}) \rightarrow \mathrm{S}\left(7 f^{2} D_{5 / 2}\right), \\
& \mathrm{S}\left(7 f^{2} D_{5 / 2}\right)+h \nu(\mathrm{UV}) \rightarrow \mathrm{S}^{+} .
\end{aligned}
$$

The detection efficiency of the $\mathrm{CO}\left(\Sigma^{+}-\Sigma^{+}, Q\right.$-branch $)$ transitions is free from the alignment factors. ${ }^{2}$ The detection efficiency of $\mathrm{S}\left({ }^{1} D\right)$ might depend on the alignment factor. ${ }^{13}$ Concentrations of OCS in $\mathrm{Ar}$ (stagnation pressure $=800$ Torr) were changed from $0.5 \%$ to $5 \%$ in order to check any effect of cluster formation. Under our experimental conditions, no effect was found in the observed images. The rotational temperature of OCS is not known precisely but can be estimated from the $7 \mathrm{~K}$ rotational temperature of $\mathrm{NO}$, which was expanded under the same conditions.

As an IR light source, the $1064 \mathrm{~nm}$ fundamental of the $\mathrm{Nd}$ :YAG laser (typically $90 \mathrm{~mJ} /$ pulse) was used to pump the electronically excited OCS. The UV and IR laser pulses were counterpropagated into the interaction region. Each laser pulse was focused with a lens $(f=20 \mathrm{~cm})$ on the pulsed molecular beam of OCS diluted in Ar.

The photofragment $\mathrm{CO}$ and $\mathrm{S}$ were detected by velocity map imaging spectroscopy. ${ }^{14,15}$ Briefly, the ions were focused onto a microchannel plate (MCP) mounted on the end of a $55 \mathrm{~cm}$ long flight tube. Electrons ejected from the rear

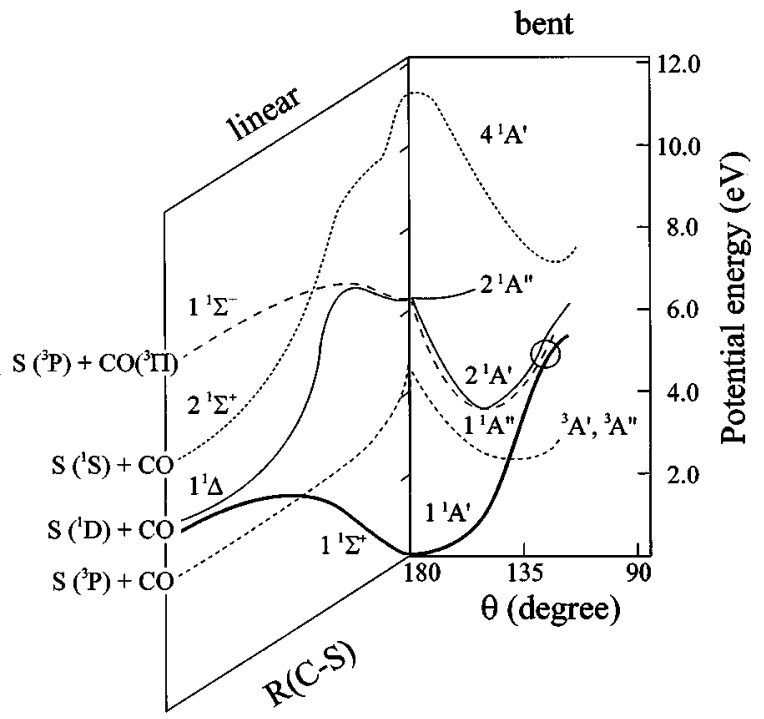

FIG. 1. Schematics of the potential energy curves of OCS as a function of bond angle and internuclear distance after Refs. 3,6,7. The circle suggests a perturbed region (see text).

plate of the MCP created an image on a phosphor screen. The 2D image was recorded by a CCD camera attached with a gated image intensifier, and was accumulated in a computer. The experimentally observed $2 \mathrm{D}$ images were backprojected by a method similar to that used in computerized tomography. The velocity distribution was obtained by the radii of the equatorial slices of the backprojected 3D images. Assuming axial recoil, the observed anisotropy parameter, $\beta$, was obtained by a least squares fit of the $3 \mathrm{D}$ slice to the angular distribution function:

$$
I(\theta)=(1 / 4 \pi)\left\{1+\beta P_{2}(\cos \theta)\right\},
$$

where $I(\theta)$ is the normalized angular distribution of the photofragment, $\theta$ is the angle between the velocity of the photofragment and the electric vector of the dissociating laser beam, and $P_{2}(x)=\left(3 x^{2}-1\right) / 2 .{ }^{16,17}$ In order to take an image the laser wavelength was scanned over an appropriate Doppler width $\left(0.15-0.3 \mathrm{~cm}^{-1}\right)$ for each $J$ level. Spectral resolution of the dye laser was about $0.2 \mathrm{~cm}^{-1}$.

\section{RESULTS}

\section{A. UV photodissociation of OCS}

\section{Pathway (1)}

Figure 2 shows equatorial slices of the photofragment images obtained as a function of rotational levels of $\operatorname{CO}\left(X^{1} \Sigma^{+}, J=45-67\right)$. Above $J \geqslant 68$, images with an appreciable signal/noise ratio could not be measured due to weak signal intensity and a small radius. From the observed images of Fig. 2, center-of-mass translational energy distributions $P\left(E_{t}\right)$ and angular anisotropy parameters $\beta$ are calculated. Table I shows the averaged values of the experimentally obtained c.m. translational energies along with the 

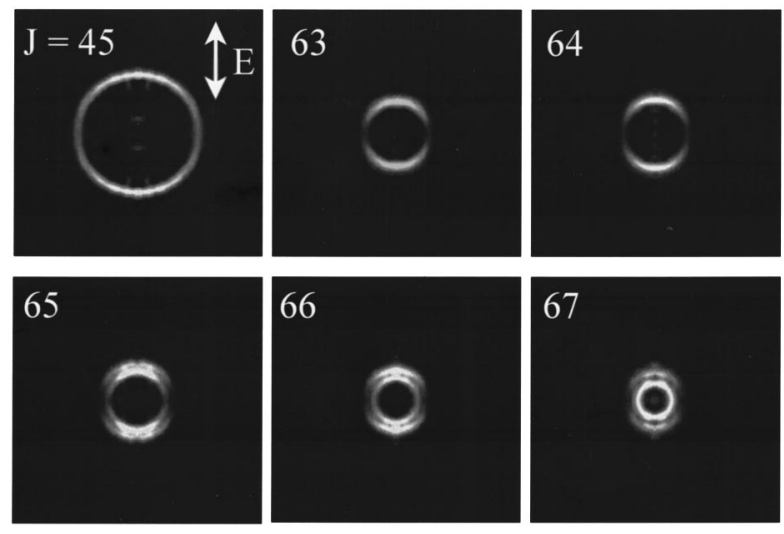

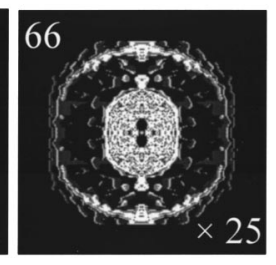

FIG. 2. Equatorial slices of the 3D images for $\mathrm{CO}\left(X^{1} \Sigma^{+}, J\right)$. In the lower right panel, $\operatorname{CO}(J=66)$ is enhanced in intensity to show the outer ring corresponding to the $\mathrm{S}\left({ }^{3} \mathrm{P}\right)$ formation. calculated translational energies for dissociation pathway (1). Considering the error margins of $\pm 0.2 \mathrm{kcal} \mathrm{mol}^{-1}$, they are in good agreement with each other. As shown in Table II, $\beta$ values increase from 0.7 to 1.2 with $J$ from 45 to 65 . These results are similar to those reported previously. ${ }^{1-3}$ Figure 3 shows $P\left(E_{t}\right)$ for various $J$ levels of CO. For example, $P\left(E_{t}\right)$ of $\mathrm{CO}(J=67)$, the arrow indicates the peak position for dissociation pathway (1). Thus, by evaluating translational energies of $\mathrm{CO}$ from the radii of the images, the singlet channel (1) is confirmed.

\section{Pathway (3)}

When the $J$ level of CO increases from 63 to 67, one notices bimodal distributions in $P\left(E_{t}\right)$ and double rings are clearly seen for $J \geqslant 65$ in the images. The energy difference between these bimodal distributions, $1.5 \mathrm{kcal} \mathrm{mol}^{-1}$, corresponds to the bend frequency of $\operatorname{OCS}\left(\nu_{2}=520 \mathrm{~cm}^{-1}\right)$. Hence, it is reasonable to assign the outer ring formation to a hot band channel (3). Even if the pulsed molecular beam is translationally and rotationally supercooled, the vibrationally hot band in the bend mode is active in the UV photodissociation. For the double rings of $\mathrm{CO}(J=67)$, the $\beta_{\text {in }}$ value of the inner ring decreases as little as zero (Table II). Since $\beta_{\text {out }}$ for the outer ring is 1.2 , this small value of $\beta_{\text {in }}$ is not caused by an instrumental resolution problem. The clear appearance of the hot band and the lowering of $\beta_{\text {in }}$ should be related to the dissociation dynamics.

TABLE I. Center-of-mass translational energies for various rotational levels of $\mathrm{CO}\left(X^{1} \Sigma^{+}, v=0, J\right)$ (Fig. 3).

\begin{tabular}{lcccccc}
\hline \hline & \multicolumn{5}{c}{$E_{t}\left(\mathrm{kcal} \mathrm{mol}^{-1}\right)^{\mathrm{a}}$} \\
\cline { 2 - 7 } & $J=45$ & 63 & 64 & 65 & 66 & 67 \\
\hline $\mathrm{OCS}(\nu=0) \rightarrow \mathrm{CO}+\mathrm{S}\left({ }^{1} D\right)$ & 13.6 & 3.7 & 3.2 & 2.4 & 2.0 & 1.3 \\
& $(14.7)$ & $(4.1)$ & $(3.5)$ & $(2.8)$ & $(2.1)$ & $(1.3)$ \\
$\mathrm{OCS}\left(\nu_{2}=1\right) \rightarrow \mathrm{CO}+\mathrm{S}\left({ }^{1} D\right)$ & $\mathrm{b}$ & 4.9 & 4.5 & 3.6 & 3.5 & 3.2 \\
$\mathrm{OCS} \rightarrow \mathrm{CO}+\mathrm{S}\left({ }^{3} P\right)$ & & $(5.6)$ & $(4.9)$ & $(4.3)$ & $(3.5)$ & $(2.8)$ \\
& & & & 27 & 32 & 33 \\
& & & & $(29)$ & $(28)$ & $(28)$ \\
\hline \hline
\end{tabular}

${ }^{a}$ Error margins are $\pm 10 \%$. Numbers in parentheses are calculated translational energies for corresponding dissociation pathways.

${ }^{b}$ Contribution of the hot band process cannot be resolved from the cold band process within the energy resolution.

\section{Pathway (2)}

When the $\mathrm{CO}(J=66)$ image is intensified as shown in the lower right hand side of Fig. 2, one notices a weak outmost ring ( $\beta_{\text {triplet }}=0.4 \pm 0.2$ ) that corresponds to triplet channel (2). Nan et al. ${ }^{5}$ reported $\beta_{\text {triplet }}=0.3 \pm 0.2$ for $\mathrm{S}\left({ }^{3} P\right)$ produced from the $222 \mathrm{~nm}$ photodissociation of OCS. This outmost ring is distinctly seen for $\mathrm{CO}(J=65-67)$ but is weak for $\mathrm{CO}(J \leqslant 64)$. Since the intersection of the singlet and triplet potential curves can occur on the bend coordinate, the triplet channel is open only for the highly bent structure of OCS, and hence, $\mathrm{CO}$ is produced in highly rotationally excited levels.

\section{B. Effect of IR irradiation on photodissociation of OCS}

Pathway(4): Figure 4 shows the $\mathrm{CO}$ images with intense IR irradiation of the YAG laser output at $1.06 \mu \mathrm{m}$ as well as UV irradiation at $\lambda_{\mathrm{UV}}=229.864 \mathrm{~nm}$. This UV wavelength corresponds to the REMPI transition centered at $\mathrm{CO}(J$ $=63$ ). The intense IR irradiation bleaches the inner CO image of singlet pathway (1) and generates the outer ring of a new pathway. In this case, UV and IR laser pulses are temporarily and spatially overlapped. Obtained $\beta=0.6 \pm 0.3$ for the outer ring is smaller than $\beta=1.4 \pm 0.1$ for the inner ring. When the direction of the IR electric vector was changed from parallel to perpendicular with respect to the detector axis, no change of the images was observed. When the UV wavelengths were shifted from the resonant wavelengths from $J=54$ to 66 , only a small change was observed in the size of the outer rings. In other words, even when the UV wavelength was not completely resonant with the REMPI transitions of $\mathrm{CO}$, additional IR photons induce the ionization process of $\mathrm{CO}$.

TABLE II. Anisotropy parameters $(\beta)$ from the photodissociation of OCS at $230 \mathrm{~nm}$ (Fig. 2).

\begin{tabular}{llcccccc}
\hline \hline & & \multicolumn{7}{c}{$\beta$} \\
\cline { 2 - 8 } & & $J=45$ & 63 & 64 & 65 & 66 & 67 \\
\hline $\mathrm{OCS}(\nu=0) \rightarrow \mathrm{CO}+\mathrm{S}\left({ }^{1} D\right)$ & $\beta_{\text {in }}$ & 0.7 & 1.4 & 1.3 & 1.2 & 0.6 & 0.0 \\
$\mathrm{OCS}\left(\nu_{2}=1\right) \rightarrow \mathrm{CO}+\mathrm{S}\left({ }^{1} D\right)$ & $\beta_{\text {out }}$ & & $\mathrm{a}$ & $\mathrm{a}$ & 1.7 & 1.6 & 1.2 \\
$\mathrm{OCS} \rightarrow \mathrm{CO}+\mathrm{S}\left({ }^{3} P\right)$ & $\beta_{\text {triplet }}$ & & & & $\mathrm{a}$ & 0.4 & 0.4 \\
\hline
\end{tabular}

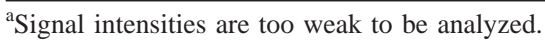




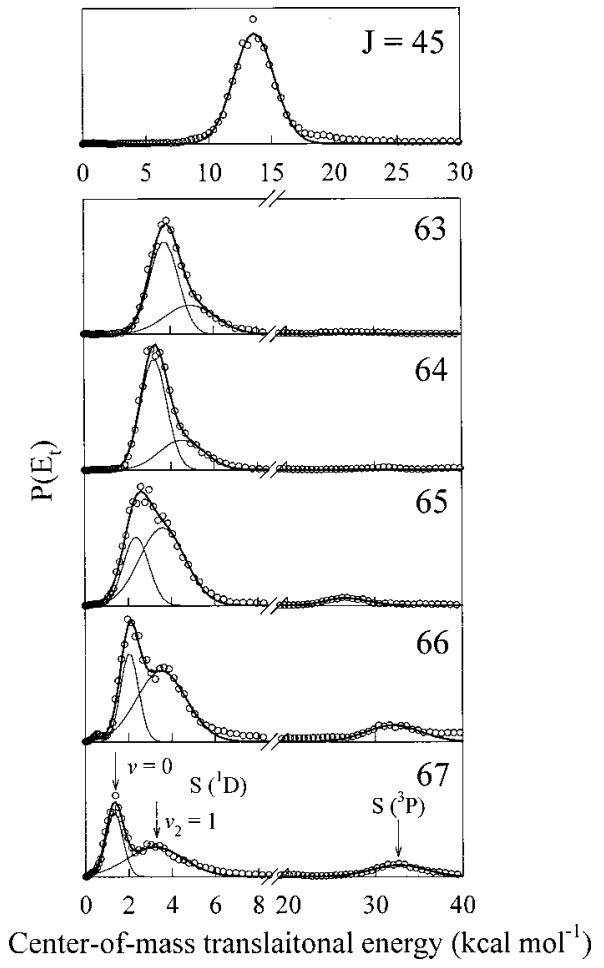

FIG. 3. Center-of-mass translational energy distribution $P\left(E_{t}\right)$ of the photofragments for various $J$ levels of $\mathrm{CO}$. The lower energy peak has two components. One is due to cold band excitation of OCS and the other to hot band excitation of $\operatorname{OCS}\left(v_{2}=1\right)$. Note that the energy scale of the topmost column is not the same as the rest.

Figure 5 shows photoexcitation spectra of the outer and inner rings, which were measured with and without an IR pulse as a function of probe (and also dissociation) laser wavelength. The spectrum of the inner ring without IR has the rotationally resolved $Q$-branch structure with slightly diffused features due to Doppler widths, which is the same as reported previously. ${ }^{2}$ However, the excitation spectrum of the outer ring with IR is decreased in intensity and is significantly different in shape from the two-photon REMPI spectrum of $\mathrm{CO}$ without IR. These results also suggest that the UV wavelengths were not completely resonant with the REMPI transitions of CO.
Since the translational energies of the outer images of $\mathrm{CO}$ cannot be attributed to any of the UV dissociation pathways $(1-3)$, the only reasonable explanation is that (a) the formation of $\mathrm{S}\left({ }^{1} S\right)$ atom after absorption of one UV and two IR photons, and (b) $\mathrm{CO}$ in the high rotational levels, $J$ $=70-80$ (centered at $\sim 74$ ), is nonresonantly ionized with $h \nu(\mathrm{UV})+2 h \nu(\mathrm{IR})$. These high $J$ levels are estimated by the translational energies of the outer rings at various UV wavelengths. Thus, $\mathrm{S}\left({ }^{1} S\right)$ and $\mathrm{CO}$ in the higher $J$ levels are produced from the dissociation of bent OCS.

Figure 4 shows the change of images as a function of the IR intensity and temporal overlap between UV and IR pulses. The intensity of the outer ring increases nonlinearly with IR intensity, in agreement with the photoexcitation mechanism involving multi-photon absorption of IR pulses. The outer ring disappears when temporal overlap is lost. These results eliminate involvement of vibrational excitation of the ground state $\operatorname{OCS}(v)$. Only a short-lived intermediate can be involved for the formation of the upper repulsive state. Hence, the photoabsorption process is a sequential multiphoton process of one UV photon followed by two IR photons. Product $\mathrm{CO}$ is ionized by a multiphoton process with UV and IR:

$$
\begin{aligned}
& \operatorname{OCS}(v=0)+h \nu(\mathrm{UV}) \rightarrow \mathrm{OCS}\left(2{ }^{1} A^{\prime}\left(1^{1} \Delta\right)\right), \\
& \operatorname{OCS}\left(2{ }^{1} A^{\prime}\left(1^{1} \Delta\right)\right)+2 h \nu(\mathrm{IR}) \\
& \quad \rightarrow \operatorname{CO}\left(X^{1} \Sigma^{+}, J \sim 74\right)+\mathrm{S}\left({ }^{1} S\right), \\
& \operatorname{CO}\left(X^{1} \Sigma^{+}, J \sim 74\right)+2 h \nu(\mathrm{UV})+n h \nu(\mathrm{IR}) \rightarrow \mathrm{CO}^{+} .
\end{aligned}
$$

\section{Probe of $S\left({ }^{1} D\right)$ atoms}

In order to investigate any change of the rotational distribution of $\mathrm{CO}$ from the dissociation pathway (1) with and without IR, the image of the counter fragment $S\left({ }^{1} D\right)$ was probed at $230.00 \mathrm{~nm}$. This UV pulse dissociates OCS by a one-color laser photodissociation scheme:

$$
\begin{aligned}
& \text { OCS }+h \nu(230.00 \mathrm{~nm}) \rightarrow \mathrm{OCS}\left(2{ }^{1} A^{\prime}\left(1^{1} \Delta\right)\right) \\
& \rightarrow \mathrm{CO}\left(X^{1} \Sigma^{+}, J\right)+\mathrm{S}\left({ }^{1} D\right), \\
& \mathrm{S}\left({ }^{1} D\right)+(2+1) h \nu \rightarrow \mathrm{S}^{+} .
\end{aligned}
$$

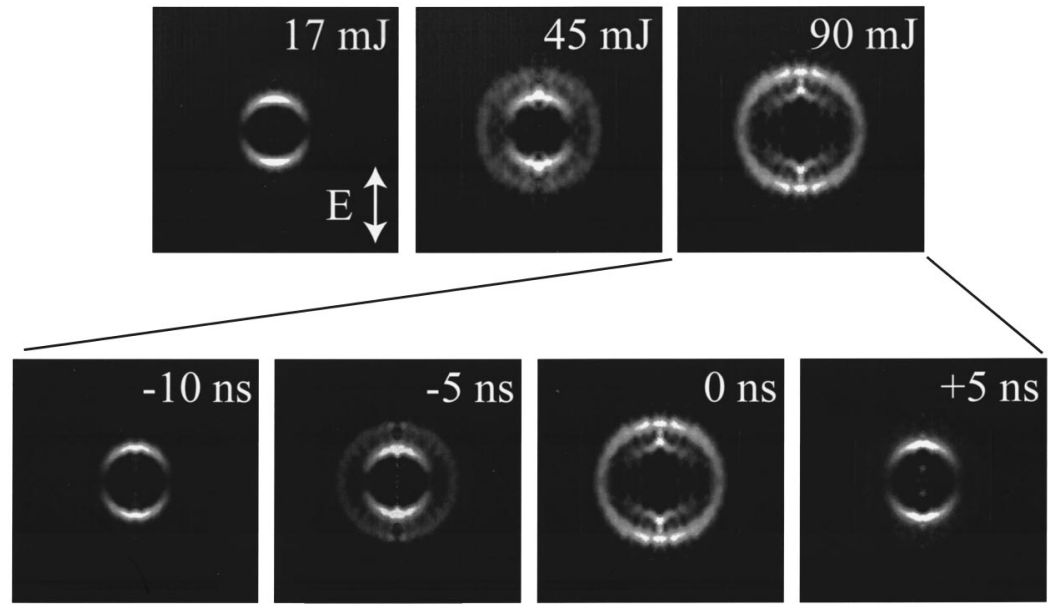

FIG. 4. Effect of intense IR pulse laser irradiation on $\mathrm{CO}(J=63)$ images. Upper panel shows effect of IR laser intensity at $1.06 \mu \mathrm{m}$. Outer ring appears for $>17$ $\mathrm{mJ}$. Lower panel shows temporal profiles of images for various delays between the IR and UV laser pulses. 


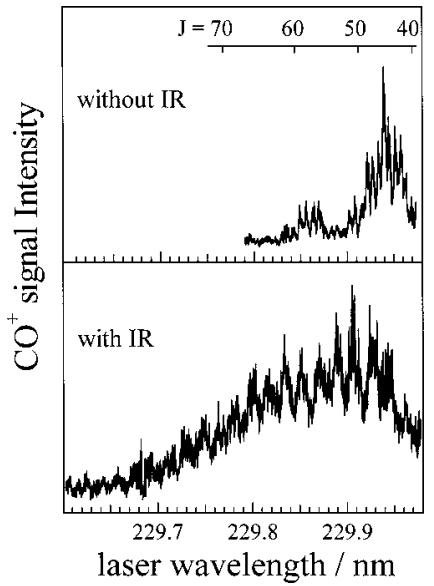

FIG. 5. Photoexcitation spectra of the outer and inner rings of CO. Upper panel shows CO produced from the OCS photodissociation in the one-color UV laser experiment without $1.06 \mu \mathrm{m}$ IR laser pulse. The structure of $Q$-branch heads is seen for the two-photon $Q$-branch transitions; $\mathrm{CO}\left(X^{1} \Sigma^{+}, J\right)+2 h \nu(229.84-229.98 \mathrm{~nm}) \rightarrow \mathrm{CO}\left(C^{1} \Sigma^{+}, J\right)$. In the lower panel, OCS is excited by UV and IR pulses, and product CO is nonresonantly ionized by UV +IR.

As shown in Fig. 6, $P\left(E_{t}\right)$ of the images have two peaks at 4.2 and $13.2 \mathrm{kcal} \mathrm{mol}^{-1}$ with the relative intensity ratio of 1 and 5, which correspond to the bimodal rotational energy distributions of $\mathrm{CO}$. Note that the hot band dissociation contributes little to this bimodal distributions. The bimodal distribution is mainly due to two different dissociation dynamics, direct and predissociation pathways, according to Suzuki et $a .^{3}$ The inner and outer rings have an observed $\beta_{\text {inner }}$ $=1.2 \pm 0.1$ and an observed $\beta_{\text {outer }}=0.33 \pm 0.03$, respectively. Due to a possible electronic orbital alignment effect of $\mathrm{S}\left({ }^{1} D\right)$, the observed $\beta$ parameters are lowered from nascent ones, but reflect qualitatively the $\beta$ values for $\mathrm{CO}$ in Table II, that is, the higher $\beta$ for the higher rotational level of $\mathrm{CO}$ and

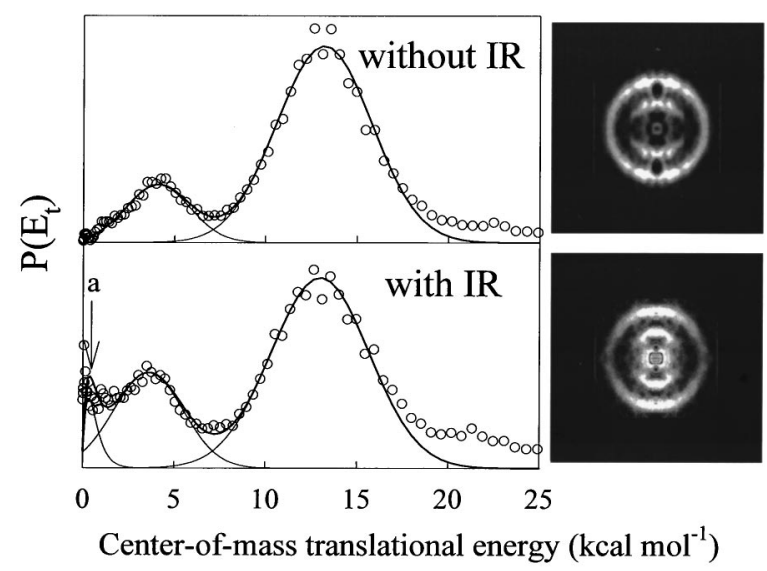

FIG. 6. Equatorial slices of the 3D images and $P\left(E_{t}\right)$ for $\mathrm{S}\left({ }^{1} D\right)$ observed from OCS photodissociation at $230.00 \mathrm{~nm}$. The double rings correspond to the bimodal rotational energy distributions of CO. Upper panel was measured with UV (0.2 mJ/pulse) and without IR laser pulse. Lower panel was measured with increasing UV laser intensity $(0.5 \mathrm{~mJ} /$ pulse $)$ and with IR (80 $\mathrm{mJ} /$ pulse). A peak marked with (a) is due to IR multiphoton dissociation, which corresponds to the central peak of the lower image. the lower $\beta$ for the lower rotational level. These results are similar to those reported for the $223 \mathrm{~nm}$ photodissociation by Mo et al. ${ }^{18}$

Now additional IR pulses were supplied to the interaction region. Because the additional IR pulses at the ordinary UV laser intensity condition decreased the signal intensity of $\mathrm{S}\left({ }^{1} D\right)$ as well as $\mathrm{CO}(J=$ high $)$, the UV laser intensity was increased to $0.5 \mathrm{~mJ} /$ pulse, about three times stronger than the ordinary intensity. Since the detection scheme is the multiphoton process listed above, the signal intensity should be increased, at least as the UV laser intensity cubed. The translational energy distribution $P\left(E_{t}\right)$ with IR thus obtained is essentially the same as that without IR. The lowest energy component near to zero in the lower panel (with IR) corresponds to the central image of $S^{+}$that is produced from IR multiphoton dissociation of OCS. About the $\beta$ values with IR, the observed $\beta$ values increases to $\beta_{\text {inner }}=1.4 \pm 0.1$ and $\beta_{\text {outer }}=0.6 \pm 0.1$, compared to those without IR. These increases may be attributable to an alignment effect by intense nonresonant IR laser pulses as will be discussed below.

\section{DISCUSSION}

\section{A. Hot band excitation and $S\left({ }^{3} P\right)$ formation}

As seen from the behavior of the potential curves OCS in Fig. 1, the bend mode plays a central role in the photoexcitation and dissociation dynamics, because it reduces the energy levels, enhances the potential curve intersection, and increases Franck-Condon overlaps between the ground and excited states at the lower excitation energy region. In the present work, the hot band channel (3) occurs for $\mathrm{CO}(J$ $\geqslant 65)$. The higher rotational levels of $\mathrm{CO}$ are selectively probed in the present experiment, which comes only from the bent OCS. At $289 \mathrm{~K}, 8 \%$ of the molecules are in $v_{2}$ $=1$. There may be somewhat less in the expanded molecular beam but it is likely that the a few percent are still in the $v_{2}=1$ level, $520 \mathrm{~cm}^{-1}$ above the ground level. Thus, even if the fraction of the $\operatorname{OCS}\left(v_{2}=1\right)$ level is small, the photoexcitation of the hot band is not negligible. This contribution is explicable in terms of the Franck-Condon factor for optical excitation from the $v_{2}=1$ level at the wavelength being much larger than that of excitation from the $v=0$ level. In addition, vibrational cooling in the molecular beam was not complete. ${ }^{19}$ This is the case for the hot band dissociation of $\mathrm{O}_{3}$ in a molecular beam. ${ }^{20,21}$ Since the electronic transition is the same as for the cold and hot band excitation, the $\beta$ values should be the same for both channels. However, as shown in Table II, the $\beta_{\text {in }}$ value for the inner ring decreases to as little as zero, while $\beta_{\text {out }}$ for the outer ring stays at 1.2. These results would suggest a strong interaction among $2{ }^{1} A^{\prime}$, $1^{1} A^{\prime \prime}$ and $1^{1} A^{\prime}$ through the bending mode near the region marked with the circle in Fig. 1. An additional explanation is "transverse recoill" dissociation mechanism, where the photofragments have little excess energy and are ejected at right angles to the molecular axis by the rotational motion of the molecule like close to the threshold for photodissociation. $^{17,22}$

Similarly, triplet pathway (2) is open mainly for the 
higher rotational levels $\mathrm{CO}(\geqslant 65)$, because the lowest ${ }^{3} A^{\prime}$ and ${ }^{3} A^{\prime \prime}$ potential curves present at $3.5-4.96 \mathrm{eV}$ can intersect with the singlet states $\operatorname{OCS}\left(2{ }^{1} A^{\prime \prime}\right.$ and $\left.2{ }^{1} A^{\prime}\right)$ in the bend coordinate. It is noteworthy that $\mathrm{O}_{3}$ dissociates through intersystem crossing to a triplet channel in the photodissociation at the longer UV region where the singlet channel is energetically almost inaccessible. ${ }^{23-25}$ As for the photodissociation of $\mathrm{N}_{2} \mathrm{O}$, nonadiabatic pathways of linear molecules occur on the bend coordinate. The driving force for the $\mathrm{N}_{2} \mathrm{O}$ thermal dissociation to $\mathrm{N}_{2}+\mathrm{O}\left({ }^{3} P\right)$ is the flow of energy into the bending vibrations. ${ }^{26}$

\section{B. $S\left({ }^{1} S\right)$ formation}

When OCS is excited simultaneously with UV and IR, $\mathrm{S}\left({ }^{1} S\right)$ and $\mathrm{CO}$ are produced via pathway $\left(4^{\prime}\right)$, that is, the electronically excited state absorbs two IR photons. The first UV excitation process of OCS is mainly OCS* $\left(2{ }^{1} A^{\prime}\left(1^{1} \Delta\right)\right)$ $\leftarrow \mathrm{OCS}\left(X^{1} \Sigma^{+}\right)$. The upper state is repulsive along the $\mathrm{C}-\mathrm{S}$ stretching coordinate, and has a short dissociation lifetime as estimated by the $\beta$ values of the photofragments. Thus, the temporal overlap of the UV and IR must be prompt for OCS* to absorb additional IR photons as seen in Fig. 4. After additional IR photons are absorbed, the excited OCS* crosses to the OCS** $\left(4^{1} A^{\prime}\left(2^{1} \Sigma^{+}\right)\right)$potential surface that correlates with $\mathrm{S}\left({ }^{1} S\right)+\mathrm{CO}$. However, according to a quantum mechanical calculation by Suzuki et al., ${ }^{3}$ the vertical excitation energy of $\mathrm{OCS}^{* *}$ from the linear ground state is $\sim 11 \mathrm{eV}$, far above the total energy of UV+2IR $(7.7 \mathrm{eV})$ as shown in Fig. 1. However, the potential energy of OCS** is lowered in bend coordinate, and the potential surface eventually intersects with $\mathrm{OCS}^{*}$ in linear geometry at an extended bond length. Since the photoprepared OCS* has a deep minimum in the bending coordinate, it is vibrationally excited after IR multiphoton absorption and results in formation of $\mathrm{S}\left({ }^{1} S\right)+\mathrm{CO}(J)$ via crossing to OCS**.

Since the reported $\beta$ value for the VUV one-photon dissociation of OCS is 1.8, almost the maximum limit for a linear molecule, the OCS** $\left(2^{1} \Sigma^{+}\right)$state has a short dissociative lifetime to generate $\mathrm{S}\left({ }^{1} S\right)+\mathrm{CO} .{ }^{10,11}$ The present $\beta$ values for the $S\left({ }^{1} S\right)$ pathway (4) is $0.6 \pm 0.3$. For simplicity, let us consider a sequential electronic excitation and dissociation process, $|f\rangle \leftarrow|i\rangle \leftarrow|g\rangle$, where $|f\rangle$ stands for OCS** $\left(4{ }^{1} A^{\prime}\left(2{ }^{1} \Sigma^{+}\right)\right),|i\rangle$ for $\operatorname{OCS}^{*}\left(2^{1} A^{\prime}\left(1^{1} \Delta\right)\right)$, and $|g\rangle$ for $\operatorname{OCS}\left(X 1^{1} \Sigma^{+}\right)$. The angular distribution of the photofragment depends on dissociation lifetimes and the nature of the electronic transitions between the ground, intermediate and final states. ${ }^{27,28}$ As a result the $\beta$ value of the product depends strongly on the dissociative lifetime of the final state and weakly on that of the intermediate state. If the sequential electronic excitation pathway is the case, it is difficult to explain the present low $\beta$ values for the $\mathrm{S}\left({ }^{1} S\right)+\mathrm{CO}$ pathway, because the final state $\operatorname{OCS}\left(4^{1} A^{\prime \prime}\left(2^{1} \Sigma^{+}\right)\right)$has a short lifetime that should result in a large $\beta$ value for the product.

An alternative explanation is a vibrational excitation mechanism of the photoprepared $\operatorname{OCS}^{*}\left(2{ }^{1} A^{\prime}\left(1^{1} \Delta\right)\right)$. This excitation process explains the absence of a polarization effect of the IR laser light on the images;

$$
\begin{aligned}
\operatorname{OCS}(v=0)+h \nu(\mathrm{UV}) & \rightarrow \mathrm{OCS}^{*}\left(2{ }^{1} A^{\prime}\left(1^{1} \Delta\right)\right), \\
\mathrm{OCS}^{*}\left(2{ }^{1} A^{\prime}\left(1^{1} \Delta\right)\right)+ & 2 h \nu(\mathrm{IR}) \\
\rightarrow & \mathrm{OCS}^{*}\left(2^{1} A^{\prime}\left(1^{1} \Delta\right), v\right), \\
\mathrm{OCS}^{*}\left(2^{1} A^{\prime}\left(1^{1} \Delta\right), v\right) & \rightarrow \mathrm{OCS}^{* *}\left(4^{1} A^{\prime}\left(2^{1} \Sigma^{+}\right)\right) \\
& \rightarrow \mathrm{CO}\left(X^{1} \Sigma^{+}\right)+\mathrm{S}\left({ }^{1} S\right) .
\end{aligned}
$$

When the IR light intensity is so strong that the upconversion rate is much larger than the dissociation rate of the lower $\operatorname{OCS}\left(2^{1} A^{\prime}\right)$ state, bleaching of the lower repulsive state is achieved. For the formation of $\mathrm{CO}\left(X^{1} \Sigma^{+}\right)+\mathrm{S}\left({ }^{1} S\right)$, the potential surface must cross over to $\operatorname{OCS} * *\left(4{ }^{1} A^{\prime}\left(2{ }^{1} \Sigma^{+}\right)\right)$ that correlates with the products. During this predissociation pathway on the bending coordinate, accompanied by a large angular momentum excitation of $\mathrm{CO}$, the recoil direction is changed, thereby, resulting in a measurable reduction of $\beta .^{29}$ Rotational motion of the parent molecule could reduce the $\beta$ value but this effect is eliminated, because a supercooled molecular beam was used in the present experiment.

\section{Molecular alignment by nonresonant IR laser}

A molecule placed in an electric field of a pulsed laser can be aligned because of the anisotropy of its polarizability tensor. ${ }^{30,31}$ This molecular alignment effect with nonresonant IR light has been reported for $\mathrm{I}_{2}, \mathrm{CH}_{3} \mathrm{I}$ and $\mathrm{C}_{6} \mathrm{H}_{5} \mathrm{I}$ and other molecules. $^{32-34}$ It should be noted that this nonresonant alignment effect is not the same as the alignmentdepolarization effect caused by resonant rovibronic excitation. ${ }^{35}$ The alignment of the ground state OCS by intense nonresonant IR laser pulses is demonstrated by change of the anisotropy of the fragments generated by polarized UV light: ${ }^{34}$

$$
\begin{aligned}
& \mathrm{OCS}+h \nu(\mathrm{IR}) \rightarrow \mathrm{OCS}(\text { aligned })+h \nu(\mathrm{IR}), \\
& \mathrm{OCS}(\text { aligned })+h \nu(\mathrm{UV}) \rightarrow \mathrm{CO}+\mathrm{S}\left({ }^{1} D\right) .
\end{aligned}
$$

The control parameter $\gamma$ of the degree of alignment for a linear molecule is:

$$
\gamma=-\left(\alpha-\alpha_{z z}\right)\left(E^{2} / 2 k T\right),
$$

where $\alpha$ is the mean polarizability, $\alpha_{z z}$ is the element of the polarizability tensor along the symmetry axis and $E$ is the rms optical electric field. The observed anisotropy parameter, $\beta_{\text {eff }}$, for $P_{2}(\cos \theta)$ in Eq. (5) is given by

$$
\beta_{\mathrm{eff}}=\beta_{0}+\gamma\left(1+2 \beta_{0} / 7-\beta_{0}^{2} / 5\right) .
$$

In this case, the electric vectors of the IR aligning and dissociating UV lasers are set parallel to each other. The angular distribution of the $\mathrm{S}\left({ }^{1} D\right)$ photofragments is fitted to Eq. (5). The value of $\beta_{0}$ without IR pulses was measured to be $\beta_{\text {inner }}=1.2 \pm 0.1$ and $\beta_{\text {outer }}=0.33 \pm 0.03$. Upon addition of the IR pulses, observed $\beta_{\text {inner }}$ and $\beta_{\text {outer }}$ increase to $1.4 \pm 0.1$ and $0.6 \pm 0.1$, respectively, as shown in Fig. 6. This increase is caused by the alignment effect due to $\alpha-\alpha_{z z}=-2.3$ $\times 10^{-24} \mathrm{~cm}^{3} \cdot{ }^{36}$ Putting $\beta_{\text {eff }}=0.6 \pm 0.1$ and $\beta_{0}=0.33$ into Eq. (6), we have $\gamma=0.3 \pm 0.1$. This $\gamma$ value is much smaller than the estimated value of 1.4 from Eq. (6) with $T=7 \mathrm{~K}$ and $E$ 
$=10^{7} \mathrm{~V} / \mathrm{cm}$. And also, the present alignment effect is not so large as observed for $\mathrm{I}_{2}$ with a fs dissociation laser. ${ }^{32,33}$ Our explanation is that the IR laser above certain intensity does not merely align but also dissociates OCS into a different channel, probably $\mathrm{S}\left({ }^{3} P\right)$. The small increase in the $\beta$ values at this high IR pulse energies is thought to originate from OCS molecules situated further from the center of the IR region of strongest focus in the interaction region. In other words, in the volume where one would expect very strong alignment and even localization, the IR laser pulse produces another pathways by the multiphoton processes, that is, formation of $\mathrm{S}\left({ }^{1} S\right)$ and $\mathrm{S}\left({ }^{3} P\right)$.

\section{SUMMARY}

By measuring the velocity distribution of the highly rotationally excited $\mathrm{CO}$ with the photofragment imaging technique, new photodissociation channels for the first UV absorption region at $\sim 230 \mathrm{~nm}$ have been investigated:

$$
\mathrm{OCS}(v=0)+h \nu(\mathrm{UV}) \rightarrow \mathrm{CO}\left(X^{1} \Sigma^{+}, J\right)+\mathrm{S}\left({ }^{3} P\right),
$$

triplet pathway

$$
\operatorname{OCS}\left(v_{2}=1\right)+h \nu(\mathrm{UV}) \rightarrow \mathrm{CO}\left(X^{1} \Sigma^{+}, J\right)+\mathrm{S}\left({ }^{1} D\right),
$$

hot band pathway

Since $\mathrm{CO}(J=$ high $)$ is produced only from bent OCS, involvement of the bend coordinate in OCS is essential for intersystem crossing for the triplet channel and enhancement of Franck-Condon overlap for the hot band channel.

Additional photoexcitation of the UV prepared dissociative state with intense infrared laser light at $1.06 \mu \mathrm{m}$ can bleach the $S\left({ }^{1} D\right)$ formation and induces selectively the $\mathrm{S}\left({ }^{1} S\right)$ formation by sequential IR multiphoton absorption via vibrational excitation process:

$$
\mathrm{OCS}+h \nu(\mathrm{UV})+2 h \nu(\mathrm{IR}) \rightarrow \mathrm{CO}\left(X^{1} \Sigma^{+}, J\right)+\mathrm{S}\left({ }^{1} S\right) .
$$

Thus, the photodissociation pathway is controlled by excitation in the bending vibration of the dissociating molecule. In addition, by the ns IR laser pulse, a part of the ground state OCS can be aligned because of the anisotropy of its polarizability tensor.

\section{ACKNOWLEDGMENTS}

M.K. thanks the Ministry of Education of Japan for a Grant-in-Aid for the priority research field, "Molecular Physical Chemistry." The Science Collaboration Program between JSPS and DGF made this collaborative research project between M.K. and K.H.G. possible.
${ }^{1}$ N. Sivakumar, G. E. Hall, P. L. Houston, J. W. Hepburn, and I. Burak, J. Chem. Phys. 88, 3692 (1988).

${ }^{2}$ Y. Sato, Y. Masumi, M. Kawasaki, K. Tsukiyama, and R. Bersohn, J. Phys. Chem. 99, 16307 (1995).

${ }^{3}$ T. Suzuki, H. Takayanagi, S. Nanbu, and M. Aoyagi, J. Chem. Phys. 109, 5778 (1998)

${ }^{4}$ T. P. Rakitzis, P. C. Samartzis, and T. N. Kitspoulos, J. Chem. Phys. 111, 10415 (1999).

${ }^{5}$ G. Nan, I. Burak, and P. Houston, Chem. Phys. Lett. 209, 383 (1993).

${ }^{6}$ J. Hijazo, M. Gonzalez, R. Sayos, and J. J. Novoa, Chem. Phys. Lett. 222, 15 (1994).

${ }^{7}$ W. M. Flicker, O. A. Mosher, and A. Kuppermann, J. Chem. Phys. 69, 3910 (1978).

${ }^{8}$ A. R. Ravishankara, G. Hancock, M. Kawasaki, and Y. Matsumi, Science 280, 60 (1998).

${ }^{9}$ G. S. Selwyn and H. S. Johnson, J. Chem. Phys. 74, 3791 (1981).

${ }^{10}$ G. Black, R. L. Sharpless, T. G. Slanger, and D. C. Lorents, J. Chem. Phys. 62, 4274 (1975).

${ }^{11}$ C. E. Strauss, G. C. McBane, P. L. Houston, I. Burak, and J. W. Hepburn, J. Chem. Phys. 90, 5364 (1989).

${ }^{12}$ C. D. Pibel, K. Ohde, and K. Yamanouchi, J. Chem. Phys. 101, 836 (1994).

${ }^{13}$ Y-X. Mo and T. Suzuki, J. Chem. Phys. 109, 4691 (1998).

${ }^{14}$ D. W. Chandler and P. L. Houston, J. Chem. Phys. 87, 1445 (1987).

${ }^{15}$ A. T. J. B. Eppink and D. H. Parker, J. Chem. Phys. 109, 4758 (1998).

${ }^{16}$ R. Bersohn and S. H. Lin, Adv. Chem. Phys. 55, 1915 (1969).

${ }^{17}$ R. N. Zare, Mol. Photochem. 4, 1 (1972).

${ }^{18}$ Y-X. Mo, H. Takayanagi, M. C. Heaven, and T. Suzuki, Phys. Rev. Lett. 77, 830 (1996).

${ }^{19}$ G. Scoles (ed.), Atomic and Molecular Beam Methods, Vol. 1 (Oxford University Press, New York, 1988).

${ }^{20}$ K. Takahashi, M. Kishigami, Y. Matsumi, M. Kawasaki, and M. N. R. Ashfold, J. Chem. Phys. 108, 7161 (1998).

${ }^{21}$ K. Takahashi, M. Kishigami, N. Taniguchi, Y. Matsumi, M. Kawasaki, and A. J. Orr-Ewing, J. Chem. Phys. 105, 5290 (1996).

${ }^{22}$ M. Kawasaki, H. Sato, A. Fukuroda, T. Kikuchi, and T. Arikawa, J. Chem. Phys. 86, 4431 (1987).

${ }^{23}$ K. Takahashi, M. Kishigami, N. Taniguchi, Y. Matsumi, and M. Kawasaki, J. Chem. Phys. 106, 6390 (1997).

${ }^{24}$ W. Denzer, G. Hancock, J. Pinot de Moira, and P. L. Tyley, Chem. Phys. Lett. 280, 496 (1997).

${ }^{25}$ P. O’Keefe, T. Ridley, S. Wang, K. P. Lawley, and R. J. Donovan, Chem. Phys. Lett. 298, 368 (1998).

${ }^{26}$ A. J. Lorquet, J. C. Lorquet, and W. Forst, Chem. Phys. 51, 253 (1980).

${ }^{27}$ R. S. Sanders and K. R. Wilson, J. Chem. Phys. 63, 4242 (1975).

${ }^{28}$ M. Kawasaki, H. Sato, T. Kikuchi, S. Kobayashi, and T. Arikawa, J. Chem. Phys. 86, 4425 (1987).

${ }^{29}$ H-P. Loock, J-Y. Cao, and C. X. W. Qian, Chem. Phys. Lett. 206, 422 (1993).

${ }^{30}$ W-S. Kim and P. M. Felker, J. Chem. Phys. 104, 1147 (1996).

${ }^{31}$ B. Friedrich and D. Herschbach, Phys. Rev. Lett. 74, 4623 (1995).

${ }^{32}$ H. Sakai, C. P. Safvan, J. J. Larsen, K. M. Hilligsoo, K. Hald, and H. Stapelfeldt, J. Chem. Phys. 110, 10235 (1999).

${ }^{33}$ J. J. Larsen, H. Sakai, C. P. Safvan, I. Wendt-Larsen, and H. Stapelfeldt, Phys. Rev. Lett. (in press).

${ }^{34}$ A. Sugita, M. Mashino, M. Kawasaki, Y. Matsumi, R. J. Gordon, and R. Bersohn, J. Chem. Phys. 112, 2164 (2000).

${ }^{35}$ J. Zhang, C. W. Riehn, M. Dulligan, and C. Wittig, J. Chem. Phys. 104, 7027 (1996).

${ }^{36} \mathrm{An}$ average value of polarizability for $\mathrm{CO}_{2}$ and $\mathrm{CS}_{2}$ is taken for OCS from Landolt-Boernstein, Molekoeln, Vol. 2 (Springer Verlag, Berlin, 1951). $\alpha$ thus obtained is $5.03 \times 10^{-24} \mathrm{~cm}^{3}$, which is in good agreement with 5.71 $\times 10^{-24} \mathrm{~cm}^{3}$ for OCS reported by A. A. Mariotto and F. Buckley, NBS(USA) Circular 537 (1953). 